\title{
THE CATEGORY OF GENERALIZED LIE GROUPS
}

\author{
BY \\ SU-SHING CHEN AND RICHARD W. YOH
}

\begin{abstract}
We consider the category $\boldsymbol{\Gamma}$ of generalized Lie groups. A generalized Lie group is a topological group $G$ such that the set $L G=\operatorname{Hom}(R, G)$ of continuous homomorphisms from the reals $R$ into $G$ has certain Lie algebra and locally convex topological vector space structures. The full subcategory $\Gamma^{r}$ of $r$-bounded ( $r$ positive real number) generalized Lie groups is shown to be left complete. The class of locally compact groups is contained in $\Gamma$. Various properties of generalized Lie groups $G$ and their locally convex topological Lie algebras $L G=\operatorname{Hom}(R, G)$ are investigated.
\end{abstract}

1. Introduction. The category $F \Gamma$ of finite dimensional Lie groups does not admit arbitrary Cartesian products. Therefore we shall enlarge the category $F \Gamma$ to the category $\Gamma$ of generalized Lie groups introduced by K. Hofmann in [11]. $\left({ }^{1}\right)$ The category $\Gamma$ contains well-known subcategories, such as the category of compact groups [11], the category of locally compact groups (see §5) and the category of finite dimensional Lie groups.

The Lie algebra $L G=\operatorname{Hom}(\mathrm{R}, G)$ of a generalized Lie group $G$ is a vital and important device just as in the case of finite dimensional Lie groups. Thus we consider the category $\Omega$ of locally convex topological Lie algebras ( $\$ 2)$. The full subcategory $\Omega^{r}$ of $r$-bounded locally convex topological Lie algebras of $\Omega$ is roughly speaking the family of all objects on whose open semiballs of radius $r$ the Campbell-Hausdorff formula holds. The full subcategory $\Gamma^{r}$ of generalized Lie groups of $\Gamma$ with Lie algebras in $\Omega^{r}$ is considered in $\$ 5$. We show that $\Omega^{r}$ and $\Gamma^{r}$ are left complete in $\S 3$ and $\S 5$ respectively. As a consequence the class of locally compact groups is contained in $\Gamma$ and any locally compact group has a Lie algebra associated with it. In §4, several basic properties of generalized Lie groups are investigated. A closed subgroup of a generalized Lie group is proved to be a generalized Lie group. However, a closed subgroup of a Banach Lie group in the sense of [1], [7], [8] and [13] is not always a Banach

Received by the editors March 5, 1973 and, in revised form, June 30, 1973.

AMS (MOS) subject classifications (1970). Primary 18A30, 22 A05.

Key words and phrases. Generalized Lie group, Lie group, Lie algebra, locally convex topological Lie algebra, category, left completeness.

$\left({ }^{1}\right)$ In order to avoid certain difficulties, Hofmann's definition in [11] is modified in this paper. The referee pointed out these difficulties to the authors.

Copyright $\odot$ 1974, Amencan Mathematical Society 
Lie group. Thus, within the category of topological groups, $\Gamma$ has certain advantages not shared by the category of Banach Lie groups in the sense of [1], [7], [8] and [13] as generalizations of finite dimensional Lie groups. A R-quotient $H$ of a topological group $G$ is a quotient group of $G$ such that the induced map from $\operatorname{Hom}(\mathbf{R}, G)$ to $\operatorname{Hom}(\mathbf{R}, H)$ is subjective. A R-quotient of a generalized Lie group is a generalized Lie group. Any closed normal subgroup $H$ of a generalized Lie group $G$ has its Lie algebra $L H$ being a closed ideal of $L G$. In $\S 6$, we consider covering groups of generalized Lie groups. Let $G$ be a connected and locally arcwise connected (Hausdorff) topological group. Then $G$ is a generalized Lie group if and only if any covering group $\widetilde{G}$ of $G$ is a generalized Lie group. Let $G$ be a connected abelian Lie group. Then $\operatorname{Hom}(\mathbf{R}, G)$ is the universal covering group of $G$. Thus, every connected abelian Lie group is the quotient $B / D$ of a Banach space $B$ by a discrete subgroup $D$.

The authors are grateful to the referee for numerous valuable suggestions and corrections of their original manuscript.

2. Locally convex topological Lie algebras. Let $L$ be a real Lie algebra. Consider the Campbell-Hausdorff formula

$$
\begin{aligned}
x \circ y & =\sum_{\substack{m>1 ; p_{i}+q_{i}>1 \\
i=1, \cdots, m}} \frac{(-1)^{m+1}(\mathrm{ad} x)^{p_{1}}(\mathrm{ad} y)^{q_{1}} \cdots(\operatorname{ad} x)^{p_{m}}(\mathrm{ad} y)^{q_{m}-1} y}{\Sigma\left(p_{i}+q_{i}\right) \Pi\left(p_{i} !\right)\left(q_{i} !\right)} \\
& =x+y+1 / 2[x, y]+\frac{1}{12}[x,[x, y]]+\cdots
\end{aligned}
$$

of the universal enveloping algebra $U L$ of $L$. Let $D_{L}$ denote the set $\{(x, y) \in L \times L \mid x \circ y \in L\}$.

Definition 1. A Lie algebra $L$ is a locally convex topological Lie algebra if there is a family of seminorms $\left\{||_{i} \mid i \in I\right\}$ defining a locally convex Hausdorff vector space topology of $L$ such that for each $i \in I,|[x, y]|_{i} \leqslant$ $C_{i}|x|_{i}|y|_{i}$ for some $C_{i} \geqslant 0$ and $D_{L}$ contains a convex set $K$ such that $(x, y) \in K$ implies $x \circ y$ converges uniformly and absolutely on $K$.

REMARK 1. Let $L$ be a Lie algebra and | | a seminorm on $L$ satisfying $|[x, y]| \leqslant C|x||y|$. Let $K=\{(x, y) \in L \times L|| x|+| y \mid \leqslant(\log 2) / C\}$. Then the Campbell-Hausdorff series is a uniform Cauchy series on $K$ relative to the uniform structure determined by $\mid \mathrm{I}$.

Definition 2. A Lie algebra $B$ is a Banach Lie algebra if $B$ is a Banach space such that $|[x, y]| \leqslant C|x||y|$ for some $C \geqslant 0$.

Proposition 1. Every Banach Lie algebra is a locally convex topological Lie algebra.

Proof. Let $B$ be a Banach Lie algebra and $N=\{x \in B|| x \mid \leqslant$ 
$(\log 2) / 2 C\}, K=N \times N$. By Remark 1 , for each $(x, y) \in K, x \circ y$ converges uniformly and absolutely on convex set $K$.

Let $B \Omega$ and $\Omega$ denote the category of Banach Lie algebras with continuous Lie algebra homomorphisms and the category of locally convex topological Lie algebras with continuous Lie algebra homomorphisms, respectively. From Proposition $1, B \Omega$ is contained in $\Omega$ fully. Let $L_{1}$ and $L_{2}$ be two objects in $\Omega$ with families of seminorms $\left\{||_{i}^{1}\right\}$ and $\left\{||_{i}^{2}\right\}$ of the same index set $I$ respectively. An isometrical Lie algebra homomorphism $\Psi: L_{1} \rightarrow L_{2}$ is a Lie algebra homomorphism such that $|x|_{i}^{1}=|\Psi(x)|_{i}^{2}$ for all $x \in L_{1}$ and $i \in I$. $L_{1}$ is said to be a subobject of $L_{2}$ if there exists an isometrical Lie algebra monomorphism from $L_{1}$ to $L_{2} . L_{1}$ is said to be a closed subobject of $L_{2}$ if the image under monomorphism is closed in $L_{2}$. Consequently, we have the following immediate result.

Proposition 2. If $L \in|\Omega|$ and $L_{1}$ is a closed Lie subalgebra of $L$, then $L_{1}$ with relative topology is a subobject of $L$ in $\Omega$.

Proposition 3. $\Omega$ is left complete.

Proof. It suffices to show that $\Omega$ has equalizers and products of small families of objects in $\Omega$. Suppose $\Phi, \Psi: L \rightarrow L^{\prime}$ in $\Omega$. Let $E=\{x \in L \mid \Phi(x)$ $=\Psi(x)\}$ which is a closed Lie subalgebra of $L$. By Proposition $2, E$ is in $\Omega$ and consequently, the inclusion map : $E \rightarrow L$ is the equalizer of $\Phi, \Psi$ in $\Omega$.

Suppose that $\left\{L_{\alpha} \mid \alpha \in A\right\}$ is a family of objects in $\Omega$. For each $\alpha \in A$, let $K_{\alpha}$ be the convex set in $D_{L_{\alpha}}$ such that for $\left(x_{\alpha}, y_{\alpha}\right) \in K_{\alpha}, x_{\alpha} \circ y_{\alpha}$ converges uniformly and absolutely on $K_{\alpha}$. Let $\Pi_{\alpha \in A} L_{\alpha}$ be the product of $\left\{L_{\alpha} \mid \alpha \in A\right\}$ in the category of topological Lie algebras. For each $\alpha \in A$, let $\left\{||_{\alpha}^{j} \mid j \in J_{\alpha}\right\}$ be the family of seminorms defining the topology of $L_{\alpha}$. Then the product topology on $\Pi_{\alpha \in A} L_{\alpha}$ is determined by the family of seminorms $\bigcup_{\alpha \in A}\left\{||_{\alpha}^{j} \mid j \in J_{\alpha}\right\}$. For each element $\left(x_{\alpha}\right)_{\alpha \in A}$ in $\Pi_{\alpha \in A} L_{\alpha},\left|\left(x_{\alpha}\right)_{\alpha \in A}\right|_{\beta}^{j}$ is defined to be $\left|x_{\beta}\right|_{\beta}^{j}$ for $\beta \in A$ and $j \in J_{\beta}$. Let $K=\Pi_{\alpha \in A} K_{\alpha}$ which can be identified with a subset in $\prod_{\alpha \in A} L_{\alpha} \times \prod_{\alpha \in A} L_{\alpha}$. With the product topology on $\Pi_{\alpha \in A} L_{\alpha}, x \circ y$ converges uniformly and absolutely on $K$ for each $(x, y) \in K$. Clearly $K$ is a convex set and the proof is completed.

Proposition 4. Suppose that $L$ is a closed ideal of a locally convex topological Lie algebra $L^{\prime}$ in $\Omega$. Then the quotient Lie algebra $L^{\prime} / L$ is in $\Omega$.

Proof. Let $\left\{||_{i} \mid i \in I\right\}$ be the family of seminorms defining the locally convex topology of $L^{\prime}$ and $q: L^{\prime} \rightarrow L^{\prime} / L$ be the quotient map. The quotient topology on $L^{\prime} / L$ is a locally convex topology determined by a family of seminorms $\left\{\|\|_{i} \mid i \in I\right\}$ which is defined as for each $i \in I$ and $q(x) \in L^{\prime} / L$, $\|q(x)\|_{i}=\inf \left\{|y|_{i} \mid q(y)=q(x)\right\}$ (see [22]). Let $K$ be the convex set in $D_{L^{\prime}}$ 
such that for $(x, y) \in K, x \circ y$ converges uniformly and absolutely. It is clear that the set

$$
K^{\prime}=\left\{(q(x), q(y)) \in L^{\prime} / L \times L^{\prime} / L \mid(x, y) \in K\right\}
$$

is convex and for $(q(x), q(y)) \in K^{\prime}, q(x) \circ q(y)$ converges uniformly and absolutely on $K^{\prime}$.

\section{The category $\Omega^{r}$.}

DEFinition 3. For each $r \in \mathbf{R}^{+}$, the category $\Omega^{r}$ of $r$-bounded locally convex topological Lie algebras is the full subcategory of $\Omega$ consisting of objects $L$ such that the convex set $K$ in $D_{L}$ contains the set

$$
\left\{(x, y) \in L \times\left. L|| x\right|_{i}<r,|y|_{i}<r \text { for all } i \in I\right\} .
$$

Proposition 5. If $L_{1}$ is a closed Lie subalgebra of $L_{2}$ which is in $\Omega^{r}$, then $L_{1}$ is in $\Omega^{r}$.

THEOREM 1. $\Omega^{r}$ is left complete for each $r \in \mathbf{R}^{+}$.

Proof. From Propositions 3 and 5, it follows that $\Omega^{r}$ has equalizer. Also it is an immediate consequence of Proposition 3 that $\Omega^{r}$ has products.

CoRollary 1. For each $r \in \mathbf{R}^{+}$, the forgetful functor $U: \Omega^{r} \rightarrow \Omega$ preserves limits.

4. Generalized Lie groups. Let $G$ be a topological group. The space $\operatorname{Hom}(\mathbf{R}, G)$ of all continuous homomorphisms from the reals $\mathbf{R}$ into $G$ has a scalar multiplication defined by $r \cdot x(s)=x(r s)$ for $x \in \operatorname{Hom}(\mathrm{R}, G)$ and $r, s \in \mathbf{R}$. We define a map exp: $\operatorname{Hom}(\mathbf{R}, G) \rightarrow G$ by $\exp (x)=x(1)$.

DEFINITION 4. A topological group $G$ is called a generalized Lie group if $L G=\operatorname{Hom}(\mathbf{R}, G)$ is a locally convex topological Lie algebra (see Definition 1) such that

(1) The map exp: $L G \rightarrow G$ is continuous,

(2) The scalar multiplication of the Lie algebra $L G$ is the scalar multiplication of $\operatorname{Hom}(\mathbf{R}, G)$,

(3) $\exp (x+y)=\lim _{n \rightarrow \infty}(\exp (x / n) \cdot \exp (y / n))^{n}$,

(4) $\exp [x, y]=\lim _{n \rightarrow \infty}(\exp (x / n) \cdot \exp (y / n) \cdot \exp (-x / n) \cdot \exp (-y / n))^{n^{2}}$,

(5) $\exp (x \circ y)=\exp x \cdot \exp y$, for $(x, y) \in K$ (of Definition 1).

Definition 5. A topological group $G$ is called a Lie group if $L G=$ $\operatorname{Hom}(\mathbf{R}, G)$ is a Banach Lie algebra (see Definition 2) such that

(1) The map exp: $\operatorname{Hom}(R, G) \rightarrow G$ is continuous,

(2) The scalar multiplication of $L G$ is the scalar multiplication of $\operatorname{Hom}(\mathrm{R}, G)$,

(3) There is an open ball $N$ around 0 in $\operatorname{Hom}(\mathbf{R}, G)$ such that $\operatorname{expl}_{N}: N \rightarrow \exp N$ is a homeomorphism onto an open neighborhood of the identity in $G$, 
(4) On the ball $N$ of (3), the Campbell-Hausdorff multiplication is defined as a continuous function from $N \times N \rightarrow L G$ such that $\exp (x \circ y)=$ $\exp x \cdot \exp y$.

Definition 6. A generalized Lie group (Lie group) morphism $\varphi$ is a topological group morphism $\varphi: G \rightarrow G^{\prime}$ such that $\operatorname{Hom}(\mathbf{R}, \varphi): \operatorname{Hom}(\mathbf{R}, G) \rightarrow$ $\operatorname{Hom}\left(\mathbf{R}, G^{\prime}\right)$ defined by $\operatorname{Hom}(\mathbf{R}, \varphi)(x)=\varphi \cdot x$ is a topological Lie algebra morphism. For simplicity, we shall denote $\operatorname{Hom}(\mathbf{R}, \varphi)$ by $\bar{\varphi}$ in the sequel.

Remark 3. Let $\varphi: G \rightarrow G^{\prime}$ be a generalized Lie group morphism. We have the following commutative diagram

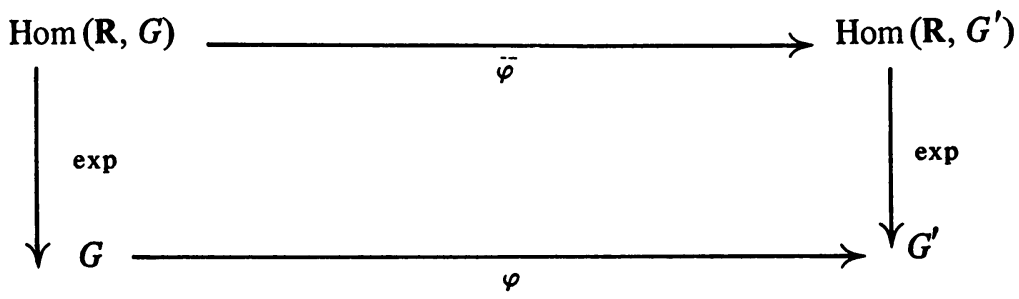

Let $\Gamma$ and $B \Gamma$ denote respectively the category of generalized Lie groups with generalized Lie group morphisms, and the category of Lie groups and Lie group morphisms. We have the following immediate result.

Proposition 7. Let $G$ be a generalized Lie group. Any closed subgroup $H$ of $G$ is a generalized Lie group.

Proof. Let $\varphi: H \rightarrow G$ be the inclusion of topological groups. Then $\bar{\varphi}: \operatorname{Hom}(\mathbf{R}, H) \rightarrow \operatorname{Hom}(\mathbf{R}, G)$ is a one to one map and we have the following commutative diagram:

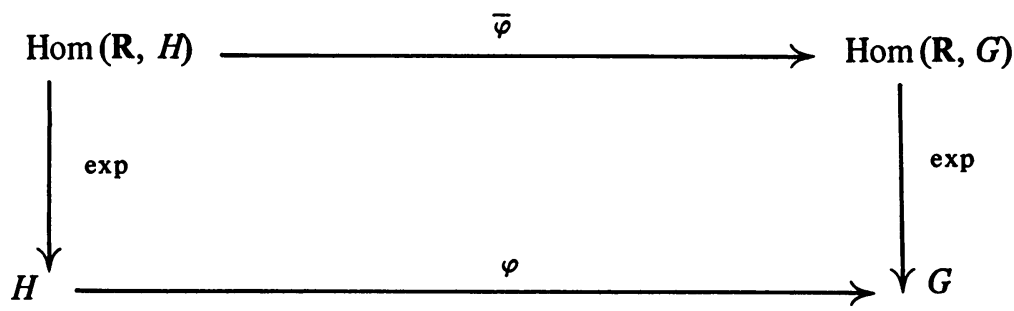

We shall show that for $x, y \in \operatorname{Hom}(\mathbf{R}, H)$, there exists $z \in \operatorname{Hom}(\mathbf{R}, H)$ such that $\bar{\varphi}(z)=\bar{\varphi}(x)+\bar{\varphi}(y)$. For notational convenience, let $\bar{\varphi}(x)=\bar{x}$ and $\bar{\varphi}(y)=\bar{y}$. We have

$$
\begin{aligned}
\exp (\bar{x}+\bar{y}) & =\lim (\exp (\bar{x} / n) \cdot \exp (\bar{y} / n))^{n}=\lim (\varphi(\exp (x / n)) \cdot \varphi(\exp (y / n)))^{n} \\
& =\lim (\varphi(\exp (x / n) \cdot \exp (y / n)))^{n} .
\end{aligned}
$$

Since $H$ is closed, we have $\lim (\exp (x / n) \cdot \exp (y / n))^{n}$ in $H$. Define $z$ : 
$\mathbf{R} \rightarrow H$ by $z(r)=\lim (\exp (r x / n) \cdot \exp (r y / n))^{n}$. Then $z(r+s)=z(r) \cdot z(s)$. In fact

$$
\begin{aligned}
\varphi(z(r+s)) & =\lim (\varphi(\exp ((r+s) / n) x)) \cdot \varphi(\exp ((r+s) / n) y)^{n} \\
& =\exp ((r+s) \bar{x}+(r+s) \bar{y})=\exp (r(\bar{x}+\bar{y})+s(\bar{x}+\bar{y})) \\
& =\varphi(z(r)) \cdot \varphi(z(s))=\varphi(z(r) \cdot z(s)) .
\end{aligned}
$$

Also we have $\bar{\varphi}(z)=\bar{x}+\bar{y}$. Since $\bar{\varphi}(z): \mathbf{R} \rightarrow G$ is continuous which is $\varphi \cdot z$, we have that $z$ is a continuous group homomorphism. Hence $z \in \operatorname{Hom}(\mathbf{R}, H)$. Similarily, $\operatorname{Hom}(\mathbf{R}, H)$ can be shown to be closed under the bracket operation [ , ] and $\bar{\varphi}$ is a Lie algebra homomorphism. Equipped with relative topology, $\operatorname{Hom}(\mathbf{R}, H)$ is a locally convex topological Lie algebra and consequently $H$ is a generalized Lie group.

The following example shows that there exists a closed subgroup of a Lie group which is not a Lie group. However from the Proposition 7, it is a generalized Lie group.

EXAMPLE. Let $G$ be the additive group of the Banach space $L=$ $L^{2}([0,1], \lambda)$ with Lebesgue measure $\lambda$. Then $G$ is an abelian Lie group with Lie algebra $L$. Let $H$ be the subgroup of all $L^{2}$-functions $f:[0,1] \rightarrow Z$ with integer values. Then $H$ is a closed, arcwise connected subgroup of $G$ without any nondegenerate one-parameter subgroup. Hence $H$ is not a Lie group.

The functor $\operatorname{Hom}(\mathbf{R},-)$ from the category of topological groups to the category of sets which takes a topological group $G$ to the set of continuous homomorphisms from $\mathbf{R}$ to $\boldsymbol{G}$ does not preserve surjectivity in general. Thus, we shall introduce the concept of R-quotient.

Definition 7. A surjective topological group homomorphism $\varphi: G \rightarrow H$ is said to be R-surjective if $\bar{\varphi}$ is surjective.

EXAMPLES. (1) Any surjective compact group homomorphism is R-surjective [11]. (2) Any surjective finite dimensional Lie group homomorphism is R-surjective.

DEFinition 8. If $\varphi: G \rightarrow H$ is R-surjective in the category of (Hausdorff) topological groups then $H$ is called a R-quotient of $G$.

Proposition 8. If $H$ is a closed normal subgroup of a generalized Lie group, then $\operatorname{Hom}(\mathbf{R}, H)$ is a closed ideal of $\operatorname{Hom}(\mathbf{R}, G)$.

Proof. According to Proposition 7, $H$ is a generalized Lie group. We need to prove that for each $y \in \operatorname{Hom}(\mathbf{R}, H)$, and $x \in \operatorname{Hom}(\mathbf{R}, G),[x, y] \in$ $\operatorname{Hom}(\mathbf{R}, H)$. Observe that

$$
\exp [x, y]=\lim \left(\exp \frac{x}{n} \cdot \exp \frac{y}{n} \cdot \exp \frac{-x}{n} \cdot \exp \frac{-y}{n}\right)^{n^{2}}
$$


is an element of $H$, by the normality of $H$. Moreover

$$
\begin{aligned}
{[x, y](r) } & =\exp r[x, y] \\
& =\lim \left(\exp \frac{\sqrt{r} x}{n} \cdot \exp \frac{\sqrt{r y}}{n} \cdot \exp \frac{-\sqrt{r x}}{n} \cdot \exp \frac{-\sqrt{r y}}{n}\right)^{n^{2}} \in H \text { for all } r \in \mathbf{R} .
\end{aligned}
$$

Thus $[x, y \mathrm{I} \in \operatorname{Hom}(\mathbf{R}, H)$. Let $\varphi: H \rightarrow G$ be the inclusion map. Suppose $N$ is a net in $\operatorname{Hom}(\mathbf{R}, H)$ such that $\bar{\varphi}(N)$ converges to $x$ in $\operatorname{Hom}(\mathbf{R}, G)$. Then $\exp (\bar{\varphi}(N))$ is a net in $G$ which converges to $\exp (x)=x(1)$. However $\exp (\bar{\varphi}(N))=\varphi(\exp (N))$ and $H$ is closed subgroup of $G$, we have $x(1) \in H$. Now for each $r \in \mathbf{R}$, the scalar multiplication of Lie algebra $r: \operatorname{Hom}(\mathbf{R}, H) \rightarrow$ $\operatorname{Hom}(\mathbf{R}, H)$ is continuous. Hence $r(N)$ is a net in $\operatorname{Hom}(\mathbf{R}, H)$ and $\bar{\varphi}(r(N))$ converges to $r x$ in $\operatorname{Hom}(\mathbf{R}, G)$. Consequently, $r x(1) \in H$. Hence $x(r) \in H$. It follows that $x \in \operatorname{Hom}(\mathbf{R}, H)$.

Proposition 9. Any R-quotient of a generalized Lie group is a generalized Lie group.

Proof. Suppose $Q$ is a R-quotient of $G$ and $q: G \rightarrow Q$ is a surjective homomorphism. Let $H$ be the kernel of $q$ which is a closed normal subgroup of $G$. By Proposition $8, \operatorname{Hom}(\mathbf{R}, H)$ is a closed ideal of Lie algebra $\operatorname{Hom}(\mathbf{R}, G)$. Since $q$ is R-quotient, we have the exact sequence

$$
0 \rightarrow \operatorname{Hom}(\mathbf{R}, H) \rightarrow \operatorname{Hom}(\mathbf{R}, G) \rightarrow \operatorname{Hom}(\mathbf{R}, Q) \rightarrow 0 .
$$

With quotient topology on $\operatorname{Hom}(\mathbf{R}, Q)$, it is a locally convex topological Lie algebra, by Proposition 4. Since $\exp \cdot \bar{q}=q \cdot \exp$ which is continuous, we have exp: $\operatorname{Hom}(\mathbf{R}, Q) \rightarrow Q$ is continuous due to the quotient topology of $\operatorname{Hom}(\mathbf{R}, Q)$. The remaining is to verify the conditions (3), (4), (5) in Definition 4.

$$
\begin{aligned}
\exp (\bar{q}(x)+\bar{q}(y)) & =\exp (\bar{q}(x+y))=q(\exp (x+y)) \\
& =q\left(\lim (\exp (x / n) \cdot \exp (y / n))^{n}\right)=\lim \left(\varphi(\exp (x / n) \cdot \exp (y / n))^{n}\right) \\
& =\lim (\exp (\bar{x} / n) \cdot \exp (\bar{y} / n))^{n} .
\end{aligned}
$$

Similarly, (4) holds. For (5), we have for $(\bar{q}(x), \bar{q}(y))$ in the convex set contained in $D_{\text {Hom }(\mathrm{R}, Q)}$,

$$
\begin{aligned}
\exp (\bar{q}(x) \circ \bar{q}(y)) & =\exp (\bar{q}(x \circ y))=q(\exp (x \circ y)) \\
& =q(\exp (x) \cdot \exp (y))=\exp (\bar{q}(x) \cdot \bar{q}(y)) .
\end{aligned}
$$

This completes the proof of Proposition 9.

Proposition 10. $\Gamma$ is left complete. 
Proof. Since the functor $\operatorname{Hom}(\mathbf{R},-)$ from the category of topological groups to the category of sets preserves limits and by Proposition 3, one can easily check $\Gamma$ has equalizers and products. Hence $\Gamma$ is left complete.

5. The category $\Gamma^{r}$.

DEFINITION 9. A generalized Lie group $G$ is said to be $r$-bounded if $L G=\operatorname{Hom}(\mathbf{R}, G)$ is in $\left|\Omega^{r}\right|$, for $r \in \mathbf{R}^{+}$.

We denote by $\Gamma^{r}$ the full subcategory of $r$-bounded generalized Lie groups of $\Gamma$, for $r \in \mathbf{R}^{+}$we have the following immediate result.

Proposition 11. Every Lie group $G$ is in $\left|\Gamma^{r}\right|$ for some $r \in \mathbf{R}^{+}$.

THEOREM 2. For each $r \in \mathbf{R}, \Gamma^{r}$ is left complete.

Proof. It is an immediate consequence of Proposition 10 and Theorem 1.

Definition 10. Let $H$ and $G$ be generalized Lie groups. $H$ is called a generalized Lie subgroup of $G$ if $H$ is a topological subgroup of $G$ and $\operatorname{Hom}(\mathbf{R}, H)$ is a subobject of $\operatorname{Hom}(\mathbf{R}, G)$ in $\Omega$.

CoRollary 2. A generalized Lie subgroup $H$ of an r-bounded generalized Lie group $G$ is $r$-bounded.

Since every continuous group homomorphism $x: \mathbf{R} \rightarrow G$ from $\mathbf{R}$ into a topological group $G$ has its image $x(\mathbf{R})$ contained in the identity component of $G$ which is a topological group denoted by $G_{0}$, we have the following immediate result.

Proposition 12. Let $G$ be a topological group and $G_{0}$ its identity component. Then the following statements are equivalent.

(1) $G_{0}$ is a generalized Lie group ( $r$-bounded),

(2) $G$ is a generalized Lie group ( $r$-bounded).

Furthermore,

(1') $G_{0}$ is a Lie group and open in $G$,

(2') $G$ is a Lie group

are equivalent.

PROPOSITION 13. Every locally compact group is a 1-bounded generalized Lie group.

Proof. By Proposition 12, it suffices to show that its identity component is a 1-bounded generalized Lie group. Let $U$ be an arbitrary neighborhood of the identity in a connected locally compact topological group $G$. By Theorem 4.6 of [18], there exists in $U$ a compact normal subgroup $H$ such that $G / H$ has no small subgroup. Hence $\operatorname{Hom}(\mathbf{R}, G / H)$ is a finite dimensional Banach Lie algebra and exp: $\operatorname{Hom}(\mathbf{R}, G / H) \rightarrow G / H$ is continuous. Moreover there is an 
open ball $N=\{x \in \operatorname{Hom}(\mathbf{R}, G / H) \mid\|x\|<r\}$ on which exp is homeomorphic and $\exp (N)$ is open in $G / H$ and $(x, y) \in K=N \times N, x \circ y$ converges uniformly and absolutely, $\exp (x \circ y)=\exp (x) \cdot \exp (y)$. Now we define a new norm | | on $\operatorname{Hom}(\mathbf{R}, G / H)$ as $|x|=r^{-1}\|x\|$ for every $x \in \operatorname{Hom}(\mathbf{R}, G / H)$ which is equivalent to \|\| . With norm | | on $\operatorname{Hom}(\mathbf{R}, G / H)$, the exp: $\operatorname{Hom}(\mathbf{R}, G / H) \rightarrow$ $G / H$ is still continuous and on the ball $N^{\prime}=\{x \in \operatorname{Hom}(\mathbf{R}, G / H)|| x \mid<1\}$, exp is homeomorphic. Moreover, for $(x, y) \in K^{\prime}=N^{\prime} \times N^{\prime}, x \circ y$ converges uniformly and absolutely and $\exp (x \circ y)=\exp (x) \cdot \exp (y)$. Consequently, $G / H$ is a 1-bounded (generalized) Lie group. It is well known that $G$ can be topologically identified with a closed subgroup of $\Pi_{\alpha \in A} G / H_{\alpha}$ where $\left\{H_{\alpha} \mid \alpha \in A\right\}$ is the family of all such compact normal subgroups of $G[18]$. Consequently by Theorem 2, Proposition 7 and Corollary 2, G is a 1-bounded generalized Lie group.

COROLlaRY 3. Any compact group is a 1-bounded generalized Lie group.

6. Covering groups of generalized Lie groups. Let $G$ be a connected and locally arcwise connected topological group and $(\widetilde{G}, G, \pi)$ be a covering space of $G$. Then $\widetilde{G}$ is a topological group and $\pi$ is an open continuous homomorphism from $\widetilde{G}$ onto $G$ with discrete kernel $D$. Moreover $D$ is contained in the center of $\widetilde{G}$. We shall show in this section that any covering group $\widetilde{G}$ of a connected, locally arcwise connected generalized Lie group is a generalized Lie group. On the other hand, when $G$ is a finite dimensional connected abelian Lie group in the classical sense, $L G$, the Lie algebra associated to $G$, is the universal covering group of $G$ which is isomorphic to $\mathbf{R}^{n}$ where $n$ is the dimension of $G$. Consequently, $G$ is isomorphic to a quotient of $\mathbf{R}^{n}$ with respect to the addition of $\mathbf{R}^{n}$. It follows that $G$ is isomorphic to $T^{m} \times \mathbf{R}^{n-m}$ where $0 \leqslant m \leqslant n$ and $T^{m}$ is a $m$-dimensional torus. We shall extend the above result to those connected Lie groups $G$ with $\operatorname{Hom}(\mathbf{R}, G)$ being abelian Banach Lie algebras which will be called connected abelian Lie groups. It turns out that a connected abelian Lie group is abelian in the group theoretic sense. Moreover $\operatorname{Hom}(\mathbf{R}, G)$ is shown to be the universal covering group of $G$. Consequently every connected abelian Lie group is the quotient $B / D$ of a Banach space $B$ where $D$ is a discrete subgroup of $B$. Thus we get a characterization of connected abelian Lie groups.

We first show two lemmas which are needed in the proof of Theorem 3. Let $C_{e}(\mathbf{R}, G)$ denote the space of continuous functions $f$ from $\mathbf{R}$ to the topological group $G$ such that $f(0)=e$, the identity of $G$, with the topology of pointwise convergence which is the relativized topology of the product topology on $G^{\mathrm{R}}$.

LEMMA 1. Let $\widetilde{G}$ with identity $\widetilde{e}$ be a covering group of a topological 
group G. Then the map $\theta: C_{e}(\mathbf{R}, G) \rightarrow C_{\widetilde{e}}(\mathbf{R}, \widetilde{G})$ defined by $\theta(f)=\widetilde{f}$ is continuous where $\widetilde{f}$ is the unique lift of $f \in C_{e}(\mathbf{R}, G)$.

Proof. Let $V$ be an open set around $\theta(f)$. We wish to find $U$ open in $C_{e}(\mathbf{R}, G)$ around $f$ such that $\theta(U) \subset V$. Since $\theta(f) \in V$, there exists an open set $W$ containing $\theta(f)$ such that $W$ is of the form $W=\{g: \mathbf{R} \rightarrow \widetilde{G} \mid$ $g(0)=\widetilde{e}$ and $g\left(r_{i}\right) \subset W_{i}$ for a neighborhood $W_{i}$ of $\theta(f)\left(r_{i}\right)$ in $\widetilde{G}$ and for finitely many $r_{i} \in \mathbf{R}$ \}. We may choose $W$ such that each $W_{i}$ is homeomorphic to $\pi\left(W_{i}\right)$ where $\pi$ is the covering map from $\widetilde{G}$ to $G$. Note that $\theta^{-1}(W)=$ $\left\{h: \mathbf{R} \rightarrow G \mid h(0)=e, h\left(r_{i}\right) \in \pi\left(W_{i}\right)\right\}$. Then $\theta^{-1}(W)$ is open in $C_{e}(\mathbf{R}, G)$ and $\theta\left(\theta^{-1}(W)\right) \subset V$.

LEMMA 2. Let $G$ be a connected and locally arcwise connected topological group and $(\widetilde{G}, G, \pi)$ be a covering group of $G$. Then there is a one to one correspondence between $\operatorname{Hom}(\mathbf{R}, G)$ and $\operatorname{Hom}(\mathbf{R}, \widetilde{G})$.

Proof. Define $\bar{\pi}: \operatorname{Hom}(\mathbf{R}, \widetilde{G}) \rightarrow \operatorname{Hom}(\mathbf{R}, G)$ as $\bar{\pi}(f)=\pi \cdot f$. By the unique lifting property, $\bar{\pi}$ is one to one. Now for each $f \in \operatorname{Hom}(\mathbf{R}, G)$, the unique lifting $\widetilde{f}$ of $f$ is a continuous group homomorphism due to the fact that $\widetilde{f}$ is a local homomorphism and $\mathbf{R}$ is simply connected. Hence $\bar{\pi}$ is onto.

THEOREM 3. Let $G$ be a connected and locally arcwise connected Hausdorff topological group. Then the following are equivalent.

(1) $G$ is a generalized Lie group.

(2) Any covering group $(\widetilde{G}, G, \pi)$ of $G$ is a generalized Lie group.

Proof. (2) $\Rightarrow(1)$. Suppose that $(\widetilde{G}, G, \pi)$ is a covering group of $G$. By Lemma $2, G$ is a R-quotient of $\widetilde{G}$. Consequently, by Proposition 9 , if $\widetilde{G}$ is generalized Lie, so is $G$.

(1) $\Rightarrow(2)$. Suppose that $G$ is a generalized Lie group and $(\widetilde{G}, G, \pi)$ is a covering group of $G$. By Lemma 2 , the map $\bar{\pi}: \operatorname{Hom}(\mathbf{R}, \widetilde{G}) \rightarrow \operatorname{Hom}(\mathbf{R}, G)$ is bijective. We shall give a topological Lie algebra structure on the set $\operatorname{Hom}(\mathbf{R}, \widetilde{G})$ such that $\bar{\pi}$ is a homeomorphic Lie algebra isomorphism. Consequently, $\operatorname{Hom}(\mathbf{R}, \widetilde{G})$ is a locally convex topological Lie algebra. We shall then show that the map $\exp : \operatorname{Hom}(\mathbf{R}, \widetilde{G}) \rightarrow \widetilde{G}$ is continuous. Note that we have $\exp \cdot \bar{\pi}=$ $\pi \cdot \exp$. For $\tilde{x} \in \operatorname{Hom}(\mathbf{R}, \widetilde{G})$ and an open set $V$ containing $\widetilde{x}(1)$ in $\widetilde{G}$, we wish to find a neighborhood $U$ of $\tilde{x}$ such that $\exp (U) \subset V$. Since $\pi$ is a covering map, there is an open neighborhood $W$ of $\tilde{x}(1)$ such that $W \subset V$ and $W$ is homeomorphic to $\pi(W)$ which is an open neighborhood of $\pi \tilde{x}(1)$. Consequently $U=\bar{\pi}^{-1} \cdot \exp ^{-1}(\pi(W))$ is an open neighborhood of $\tilde{x}$ in $\operatorname{Hom}(\mathbf{R}, \widetilde{G})$ and $\exp (U) \subset V$. We now show 


$$
\exp (\tilde{x}+\tilde{y})=\lim \left(\exp \frac{\tilde{x}}{n} \cdot \exp \frac{\tilde{y}}{n}\right)^{n}
$$

and

$$
\exp ([\tilde{x}, \tilde{y}])=\lim \left(\exp \frac{\tilde{x}}{n} \cdot \exp \frac{\tilde{y}}{n} \cdot \exp -\frac{\tilde{x}}{n} \cdot \exp -\frac{\tilde{y}}{n}\right)^{n^{2}}
$$

Define functions $(\tilde{x} / n \cdot \tilde{y} / n)^{n}: \mathbf{R} \rightarrow \widetilde{G}$ as $\left.(\tilde{x} / n \cdot \tilde{y} / n)^{n}(r)=(\tilde{x} / n)(r) \cdot(\tilde{y} / n)(r)\right)^{n}$ for each $r \in \mathbf{R}$ and each $n \in Z^{+}$which are continuous functions. Similarily, define continuous functions $(x / n \cdot y / n)^{n}: \mathbf{R} \rightarrow G$ by $(x / n \cdot y / n)^{n}(r)=$ $(x / n)(r) \cdot(y / n)(r)$ for each $r \subset \mathbf{R}$ and each $n \in Z^{+}$. Since

$$
\lim (\exp x / n \cdot \exp y / n)^{n}=\exp (x+y),
$$

we have for each $r \in \mathbf{R}, \lim (\exp r x / n \cdot \exp r y / n)^{n}=\exp (r x+r y)$ which is $\lim ((x / n)(r) \cdot(y / n)(r))=(x+y)(r)$. Consequently, the sequence of continuous functions $\left\{(x / n \cdot y / n)^{n}\right\}$ converges pointwise to $x+y$. By Lemma 1, the sequence of continuous functions $\left\{(\tilde{x} / n \cdot \tilde{y} / n)^{n}\right\}$ converges pointwise to $\tilde{x}+\tilde{y}$. In particular, $\lim ((\tilde{x} / n)(1) \cdot(\tilde{y} / n)(1))^{n}=(\tilde{x}+\tilde{y})(1)$ holds, which says

$$
\lim (\exp \tilde{x} / n \cdot \exp \tilde{y} / n)^{n}=\exp (\tilde{x}+\tilde{y}) .
$$

Similarly, we can show

$$
\exp ([\tilde{x}, \tilde{y}])=\lim \left(\exp \frac{\tilde{x}}{n} \cdot \exp \frac{\tilde{y}}{n} \cdot \exp \left(-\frac{\tilde{x}}{n}\right) \cdot \exp \left(-\frac{\tilde{y}}{n}\right)\right)^{n^{2}} \cdot
$$

Finally, we show over some convex set $\widetilde{K},(\widetilde{x}, \widetilde{y}) \in \widetilde{K}$ implies $\widetilde{x} \circ \tilde{y}$ converges uniformly and absolutely and $\exp (\tilde{x} \circ \tilde{y})=\exp \tilde{x} \cdot \exp \tilde{y}$. Let $K$ be the convex set in $\operatorname{Hom}(\mathbf{R}, G) \times \operatorname{Hom}(\mathbf{R}, G)$ such that for $(x, y) \in K, x \circ y$ converges uniformly and absolutely and $\exp (x \circ y)=\exp x \cdot \exp y$. Then $\widetilde{K}=$ $\{(\widetilde{x}, \tilde{y}) \in \operatorname{Hom}(\mathbf{R}, \widetilde{G}) \mid(\bar{\pi}(\widetilde{x}), \bar{\pi}(\widetilde{y})) \in K\}$ is a convex set and for $(\widetilde{x}, \widetilde{y}) \in \widetilde{K}$, $\tilde{x} \circ \tilde{y}$ converges uniformly and absolutely. Let $U$ be a neighborhood of $e$ in $G$ such that $U$ is homeomorphic to the connected component $\widetilde{U}$ of $\pi^{-1}(U)$ containing $\tilde{e}$. Furthermore, we can find a symmetric neighborhood $W$ in $U$ such that $W W \subset U$ and $\widetilde{W} \widetilde{W} \subset \widetilde{U}$. Since $\widetilde{W}$ is a neighborhood of $\widetilde{\boldsymbol{e}}, \exp ^{-1}(\widetilde{W})$ is a neighborhood of 0 in $\operatorname{Hom}(\mathbf{R}, \widetilde{G})$ which contains a basic neighborhood of 0 ,

$$
V=\left\{\left.\widetilde{x} \in \operatorname{Hom}(\mathbf{R}, \widetilde{G})|| \bar{\pi}(\widetilde{x})\right|_{k} \leqslant r_{k} \text { for finitely many } k \in I\right\} .
$$

Consequently $\exp (V) \subset \widetilde{W}$. Let $\widetilde{K}=\widetilde{K} \cap(V \times V)$ which is a convex set. For $(\widetilde{x}, \widetilde{y}) \in \widetilde{K}, \widetilde{x} \circ \widetilde{y}$ converges uniformly and absolutely and 


$$
\begin{gathered}
\exp \tilde{x} \cdot \exp \tilde{y} \in \widetilde{W} \cdot \widetilde{W} \subset \widetilde{U} \text { and } \\
\exp \tilde{x} \cdot \exp \tilde{y}=\pi^{-1}(\exp x \cdot \exp y)=\pi^{-1}(\exp (x \circ y)) .
\end{gathered}
$$

On the other hand, since $\exp (x \circ y)=\pi(\exp (\tilde{x} \circ \tilde{y})) \in U$ and $\tilde{x} \circ \tilde{y}$ passes through $\tilde{\boldsymbol{e}}$, we conclude that $\exp (\tilde{\boldsymbol{x}} \circ \tilde{y}) \in U$. Consequently,

$$
\exp (\tilde{x} \circ \tilde{y})=\exp \tilde{x} \cdot \exp \tilde{y} .
$$

This completes the proof of Theorem 3.

COROllary 3. If $G$ is a connected and locally arcwise connected $r$ bounded generalized Lie group, then any covering group of $G$ is a $t$-bounded generalized Lie group for some $t \in \mathbf{R}^{+}$.

THEOREM 4. Let $G$ be a connected abelian Lie group; i.e., $\operatorname{Hom}(\mathbf{R}, G)$ is an abelian Lie algebra. Then $\operatorname{Hom}(\mathbf{R}, G)$ is the universal covering group of $G$.

Proof. Since a Lie group $G$ has a neighborhood of the identity $e$ which is homeomorphic to a ball around 0 in $\operatorname{Hom}(\mathbf{R}, G), G$ is locally arcwise simply connected. Consequently, the universal covering group $(\widetilde{G}, G, \pi)$ of $G$ exists. Since exp: $\operatorname{Hom}(R, G) \rightarrow G$ is continuous, we have a unique lifting $\varphi$ : $\operatorname{Hom}(\mathbf{R}, G) \rightarrow \widetilde{G}$ such that the following diagram commutes.

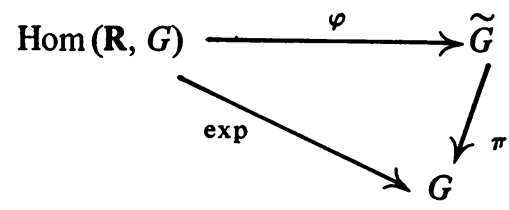

Since $G$ is an abelian Lie group, we have

$$
x \circ y=x+y \text { for all } x, y \in \operatorname{Hom}(\mathbf{R}, G) .
$$

Moreover there is a ball $N$ around 0 in $\operatorname{Hom}(\mathbf{R}, G)$ homeomorphic to $\exp (N)$ and for $x, y \in N, \exp x \cdot \exp y=\exp (x \circ y)=\exp (x+y)$. Hence exp is a local homomorphism. It follows that exp is a homomorphism which is due to the fact that $\operatorname{Hom}(R, G)$ is a simply connected group with respect to the addition. Since $(\widetilde{G}, G, \pi)$ is a covering group of $G$, there is an open neighborhood $U$ of $e$ in $G$ homeomorphic to an open neighborhood $\widetilde{U}$ of $\tilde{e}$ in $\widetilde{G}$. It follows that $U \cap \exp (N)$ is a neighborhood of $e$ and $W=\widetilde{U} \cap \pi^{-1}(\exp (N) \cap U)$ is a neighborhood of $\tilde{\boldsymbol{e}}$ in $\widetilde{G}$. Moreover $\left.\exp ^{-1} \cdot \pi\right|_{W}: W \rightarrow N$ is a local homomorphism. Since $\widetilde{G}$ is simply connected, 
$\left.\exp ^{-1} \cdot \pi\right|_{W}$ can be extended to a continuous global group homomorphism $\varphi^{\prime}$ : $\widetilde{G} \rightarrow \operatorname{Hom}(\mathbf{R}, G)$. Since $\varphi \cdot \varphi^{\prime}$ and $\varphi^{\prime} \cdot \varphi$ are local identity maps, we conclude $\varphi \varphi^{\prime}=1, \varphi^{\prime} \varphi=1$. Consequently, $\operatorname{Hom}(\mathbf{R}, G)$ is the universal covering of $G$.

COROLlARY 4. Every connected abelian Lie group is an abelian group and is the quotient $B / D$ of a Banach space $B$ by a discrete subgroup $D$ with respect to the addition.

\section{REFERENCES}

1. G. Birkhoff, Analytical groups, Trans. Amer. Math Soc. 43 (1938), 61-101.

2. S. Chen and S. Morris, Varieties of topological groups generated by Lie groups, Proc. Edinburgh Math. Soc. 18 (1972), 49-54.

3. S. Chen and R. W. Yoh, Varieties of generalized Lie groups, Colloq. Math. (to appear).

4. C. Chevalley, Theory of Lie groups. I, Princeton Math. Series, vol. 8, Princeton Univ. Press, Princeton, N. J., 1946. MR 7, 412.

5. P. M. Cohn, Lie groups, Cambridge Tracts in Math. and Math. Phys., no. 46, Cambridge Univ. Press, New York, 1957. MR 21 \#2702.

6. - Universal algebra, Harper and Row, New York, 1965. MR 31 \#224.

7. E. B. Dynkin, Normed Lie algebras and analytic groups, Uspehi Mat. Nauk 5 (1950), no. 1 (35), 135-186; English transl., Amer. Math. Soc. Transl. (1) 9 (1962), 470-534. MR 11, 712.

8. J. Eells, Jr., A setting for global analysis, Bull. Amer. Math. Soc. 72 (1966), 751 807. MR 34 \#3590.

9. S. Helgason, Differential geometry and symmetric spaces, Pure and Appl. Math., vol. 12, Academic Press, New York, 1962. MR 26 \#2986.

10. G. Hochschild, The structure of Lie groups, Holden-Day, San Francisco, London and Amsterdam, 1965. MR 34 \#7696.

11. K. Hofmann, Introduction to the theory of compact groups, Tulane Univ. Lecture Notes, 1968.

12. N. Jacobson, Lie algebras, Interscience Tracts in Pure and Appl. Math., no. 10, Interscience, New York, 1962. MR 26 \#1345.

13. R. K. Lashof, Lie algebras of locally compact groups, Pacific J. Math. 7 (1957), 1145-1162. MR 19, 1064.

14. D. Laugwitz, Über unendliche kontinuierliche Gruppen. I. Grundlagen der Theorie; Untergruppen, Math. Ann, 130 (1955), 337-350. MR 17, 762. , Über unendliche kontinuierliche Gruppen. II. Strukturtheorie lokalBanachscher Gruppen, Bayer. Akad. Wiss. Math.-Nat. K1. S.-B. 1956, 261-286. (1957). MR 19, 753.

15. M. Lazard and J. Tits, Domaines d'injectivité de l'application exponentielle, Topology 4 (1965/66), 315-322. MR 32 \#2518.

16. B. Maissen, Lie-Gruppen mit Banachrdumen als Parameterräume, Acta Math. 108 (1962), 229-270. MR 26 \#262.

17. B. Mitchell, Theory of categories, Pure and Appl. Math., vol. 17, Academic Press, New York, 1965. MR 34 \#2647.

18. D. Montgomery and L. Zippin, Topological transformation groups, Interscience, New York, 1955. MR 17, 383. 
19. G. D. Mostow, Lectures on Lie groups and Lie algebras, Yale University Lecture Notes.

20. Séminaire "Sophus Lie" de l'École Normale Supérieure, 1954/55, Théorie des algèbres de Lie, Topologie des groupes de Lie, Secrétariat mathématique, Paris, 1955. MR 17, 384.

21. . J.-P. Serre, Lie algebras and Lie groups, Lectures given at Harvard University, 1964, Benjamin, New York, 1965. MR 36 \#1582.

22. F. Treves, Topological vector spaces, distributions and kernels, Academic Press, New York, 1967. MR 37 \#726.

DEPARTMENT OF MATHEMATICS, UNIVERSITY OF FLORIDA, GAINESVILLE, FLORIDA 32601 